\title{
Balancing Security and Privacy in Genomic Range Queries
}

\author{
Xuhua Ding \\ xhding@smu.edu.sg \\ Singapore Management University
}

\author{
Ercan Ozturk \\ ercano@uci.edu \\ University of California, Irvine
}

\author{
Gene Tsudik \\ gene.tsudik@uci.edu \\ University of California, Irvine
}

\begin{abstract}
Recent spectacular advances in genome sequencing coupled with greatly reduced storage and computation costs, make genomic testing increasingly accessible to individuals. Today one can easily get his/her DNA digitized by a sequencing lab and store the result on a local device before performing a range of tests by engaging with a testing facility. Due to the inherent sensitivity of genetic material and often proprietary tests, privacy is the natural and key issue. However, heretofore insufficient attention paid to genomic security might have grave consequences, such as incorrect drug prescriptions or erroneous parentage outcomes.

Unfortunately, in genomic testing privacy and security are at odds with each other. In this paper, we reconcile security with privacy in a particular setting of genomic range queries. We do so by designing a novel technique in the form of a secure and private sparse-set range query between genomic testing facilities and individuals. The proposed technique maintains authenticity and completeness of user-supplied genomic material, while maintaining its privacy by releasing only the minimum thereof. Our experiments show that this approach is highly practical in terms of performance, for all parties involved.
\end{abstract}

\section{KEYWORDS}

genomic security, genomic privacy, range queries, range completeness

\section{ACM Reference Format:}

Xuhua Ding, Ercan Ozturk, and Gene Tsudik. 2019. Balancing Security and Privacy in Genomic Range Queries. In 18th Workshop on Privacy in the Electronic Society (WPES'19), November 11, 2019, London, United Kingdom. ACM, New York, NY, USA, 5 pages. https://doi.org/10.1145/3338498.3358652

\section{INTRODUCTION}

Technical improvements in DNA sequencing technology [22, 25, 32] and reduced costs have paved the way for ubiquitous and affordable genomic testing. Due to the private nature of the test specifics and genomic material, genomic privacy justifiably attracted much attention from the research community and numerous privacy techniques have been proposed [6,7, 9, 10, 16, 17, 28, 33, 35].

However, genomic security, even though equally important, received considerably less attention. In particular, authenticity and integrity of genomic data are often dismissed or over-simplified,

Permission to make digital or hard copies of all or part of this work for personal or classroom use is granted without fee provided that copies are not made or distributed for profit or commercial advantage and that copies bear this notice and the full citation on the first page. Copyrights for components of this work owned by others than ACM must be honored. Abstracting with credit is permitted. To copy otherwise, or republish, to post on servers or to redistribute to lists, requires prior specific permission and/or a fee. Request permissions from permissions@acm.org.

WPES'19, November 11, 2019, London, United Kingdom

(C) 2019 Association for Computing Machinery.

ACM ISBN 978-1-4503-6830-8/19/11 . \$ \$15.00

https://doi.org/10.1145/3338498.3358652 even though they are crucial to the accurate outcome of genomic tests. An erroneous (whether maliciously caused or not) genomic test result can translate into grave health risks when used for medical diagnosis, or social and family risks when used for determining familial relationships.

In this paper, we focus on efficiently reconciling security and privacy in the context of genomic range queries. Specifically, we propose a technique for secure and private genomic testing using a combination of established cryptographic tools. Our contribution is two-fold:

- We propose a novel secure and private technique for genomic range queries that can be used as a building block in various protocols and genomic representations.

- We report on a prototype implementation and evaluation of the proposed technique.

Organization. Related work is summarized Section 2, and background material is presented in Section 3. Section 4 outlines our system and security models. Section 5 describes secure and private range queries. Section 6 describes the new technique, followed by its implementation and evaluation in Sections 7 and 8, respectively. Sections 9 concludes the paper.

NOTE: Our notations are summarized in Table 1.

\section{RELATED WORK}

Prior related work focused mainly on privacy aspects of genomic tests $[5,21]$. Cryptographic techniques for providing genomic privacy included Private Set Intersection (PSI) [16, 18, 19, 23, 27], homomorphic encryption [7, 8, 15, 28] and oblivious automata [35].

Earlier examples of range proofs are [13, 14, 31]. They can be used to prove that a secret value lies in an expanded range. Boudot [11] proposed two efficient range proof protocols one of which we use in this work.

Lastly, range query completeness was explored for outsourced databases. Pang et al. [34] developed methods based on continual linking of elements, similar to techniques proposed in [30]. Hore et al., [26] focused on minimizing privacy leakage in data attributes. Li et al. [30] used Merkle hash and $\mathrm{B}^{+}$trees, and aggregated signatures to provide authenticity and integrity (with less strict privacy requirements compared to [34]). We refer to Bradley et al. [12] for a comparison of these techniques.

\section{BACKGROUND}

This section overviews genomics basics and commitment techniques. It can be skipped with no loss of continuity.

\subsection{Genomics Background}

Human DNA consists of around 3.2 billion bases (each is one of: [A]denine, [C]ytosine, [G]uanine, and [T]hymine) and only about $0.1 \%$ of DNA differs between any two individuals. Although it is not 
yet known exactly where these differences occur, many types of mutations can be used to identify an individual and determine susceptibility to diseases and/or sensitivity to drugs. Single-Nucleotide Polymorphism (SNP) is a type of genetic mutation representing a change in the nucleotide, such as $[A] \rightarrow[C]$.

Although a fully digitized raw human genome may take up to 200 Gbytes, due to relative sparsity of SNPs, a reference genome can be used to reduce storage and computation costs. In this paper, we represent DNA as an array of mutations, each of the form: $\mathbf{V}_{\mathbf{i}}=\left\{\operatorname{pos}, \mathrm{L}_{\text {pos }}\right\}$, where $i$ is the index of the mutation in the array, pos is its position in the DNA (denoted as $V_{i} . p o s$ ) and $L_{\text {pos }}$ is the base letter at position pos.

\subsection{Commitments}

A cryptographic commitment scheme is a two-phase protocol (commit and reveal) between a prover and a verifier, where the underlying commitment is hiding and binding.

In this paper, we use two types of commitments: Fujisaki-Okamoto and those based on secure cryptographic hash functions. A FujisakiOkamoto commitment [24] is of the form: $\operatorname{COM}(z)=g^{z} h^{r} \bmod n$, where $z$ is the prover-chosen secret value and $n$ is a large composite integer with factorization unknown to either party. Also, $g$ is an element with a large order in $\mathbb{Z}_{n}^{*}, h$ is an element with a large order in the group generated by $g$. The discrete logarithms of $g$ to $h$ and $h$ to $g$ are also unknown to either party. The value $r$ is randomly chosen by the prover from $\left[-2^{s} n-1,2^{s} n+1\right]$, where $s$ is a security parameter. This commitment statistically does not reveal any information to the verifier.

Commitments based on secure cryptographic hash functions offer hiding and binding if they are used with sufficiently large random salts. A hash function based commitment is of the form: $\mathbf{H}(\mathbf{z} \mid \mathbf{r})$, where $H$ is a cryptographically-secure hash function (e.g., SHA256), $z$ is the committed value, 'l' denotes concatenation and $r$ is a sufficiently long random bitstring used as a salt.

\subsection{Range Proofs}

A range proof convinces a verifier that a value is in a given range without revealing the actual value. We consider two range proof protocols from [11]. The first proves that a hidden integer $z \in$ $[x-\theta, y+\theta]$ rather than in $[x, y]$ where $\theta=2^{t+l+1} \sqrt{y-x}$ and $t$ and $l$ are security parameters. This protocol does not work well with small ranges. (Recall that DNA contains around $3.2 \times 10^{9}$ letters). The second protocol is similar, however, the secret value is first expanded to $z^{\prime}=z \cdot 2^{T}$. With a carefully chosen $T$ as $2(t+$ $l+1)+|y-x|$, this protocol's expansion rate $\delta=1$. This protocol uses two subproofs: $z-x \geq 0$ and $y-z \geq 0$, and rewrites $z-x$ and $y-z$ as the sum of a square (largest less than $z-x$ ) and a bounded number (using [14]). These subproofs jointly show that the secret value is in the requested range.

\section{SYSTEM \& SECURITY MODELS}

\subsection{System Model}

Our system model consists of an individual (Alice), a sequencing lab $(S L)$, and a tester $(T)$. Alice first obtains her digitized signed SNP representation from $S L$ - a regulated and trusted sequencing lab capable of digitizing genetic material. Modern $S L$ examples

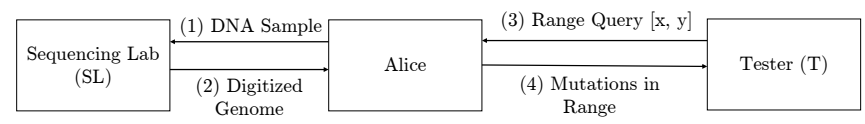

Figure 1: A Genomic Test Scenario with Order of Interactions

include hospitals and direct-to-customer (DTC) service providers. $T$ needs to perform a genomic range test on Alice's genome. The test includes one or more queries sent to Alice. Each query is in the form of two integers corresponding to low and high positions in the genome. Upon receiving the query, Alice replies with relevant information, as shown in Figure 1.

\subsection{Security Model}

In our security model, $S L$ is fully trusted by both Alice and $T$. Nonetheless, Alice is not trusted by $T$, since she may want to provide altered genomic data, for various reasons. Alice may cheat in several ways: She may report modified data to $T$, fake data at a particular position, exclude data from a particular position, or a hybrid thereof. We consider $T$ to be an honest-but-curious entity. It follows the protocol by issuing authorized queries. However, it aims to learn more information from Alice's genomic data than what the test requires. We suppose that queried ranges are pre-approved by some authority, e.g., via a public signed white-list of legitimate ranges for certain genomic tests.

\section{RANGE QUERY FORMULATION}

We denote $T$ 's query as $Q=[x, y]$, where $x$ and $y$ are locations which indicate that $T$ needs to obtain all of Alice's genomic mutations in between. Without loss of generality, suppose that Alice has $n$ mutations in the reference-based representation. We denote them as: $\mathcal{V}^{*}=\left\langle V_{1}, V_{2}, \cdots, V_{n}\right\rangle$. If Alice and $T$ trust each other, she would only reveal $\mathcal{V}^{*}$ to $T$.

We propose a secure and private range query scheme consisting of a query response function run by Alice and a verification function run by $T$. The former is denoted as $\operatorname{Resp}(Q)$ which takes a query $Q$ as input and outputs a result $\left(\mathcal{V}=\left\langle\left\{V_{i \ldots j}\right\}\right\rangle, \sigma\right)$. The latter is denoted as $\operatorname{Verfy}(\mathcal{V}, \sigma)$ and it outputs either $\mathcal{V}$ or $\perp$, depending on the verification outcome.

(1) Authenticity. All mutations reported by Alice must be authentic and not tampered with. More precisely, $\forall V \in \mathcal{V}, V \in$ $\mathcal{V}^{*}$. Note that $V$ reflects the mutation position. Hence, if $\mathcal{V}$ satisfies authenticity, it is trivial for $T$ to check its validity, i.e., whether the reported mutation is between $x$ and $y$. In other words, authenticity implies soundness.

(2) Completeness. All mutations in $\mathcal{V}$ are reported by Alice. Namely, $\forall V \in \mathcal{V}^{*}, V \in \mathcal{V}$.

(3) Privacy. There should be no information leakage on mutations other than those $T$ is allowed to learn. Specifically, $\forall V \in \mathcal{V}, \operatorname{Pr}\left(V \mid \mathcal{V}^{*}\right)=\operatorname{Pr}(V \mid \mathcal{V})$.

\section{PROPOSED CONSTRUCTION}

We now discuss several intuitive approaches to the range query problem described in Section 5 and compare them along several 
Table 1: Notation \& Descriptions

\begin{tabular}{|l|l|}
\hline Notation & Description \\
\hline Alice & Individual with a digitized genome issued by a certified sequencing lab. \\
\hline$S L$ & Sequencing Lab capable of sequencing genetic material \\
\hline$T$ & A Tester that perform genomic tests \\
\hline$Q$ & A range query with boundaries $[x, y]$ \\
\hline $\mathcal{V}^{*}$ & A list of mutations that describe Alice's DNA, used in $\Gamma$ \\
\hline$\Gamma$ & Proposed secure and private range query scheme \\
\hline $\operatorname{Resp}(Q)$ & Response function of $\Gamma$, run by Alice and outputs a response $\left(\mathcal{V}=\left\langle\left\{V_{i \ldots j}\right\}\right\rangle, \sigma\right)$ \\
\hline $\operatorname{Verfy}(\mathcal{V}, \sigma)$ & Verify function of $\Gamma$, run by $T$ and outputs $\perp$ if any verification function fails. \\
\hline $\mathrm{ZK}(z: R(z))$ & Generates a zero-knowledge proof of knowledge of $z$ such that $R(z)$ holds. \\
\hline$Z$ ZK_Verify $(p, q, r)$ & $\begin{array}{l}\text { Verifies the correctness of a zero-knowledge proof } p \text { with a commitment } q, \\
\text { such that for the committed value the predicate } r \text { holds. }\end{array}$ \\
\hline $\operatorname{Sign}_{S K_{s}}(t)$ & Signs $t$ using secret key of $s S K_{s}$ \\
\hline $\operatorname{Verify}_{P K_{s}}(t, u)$ & Verifies the signature $u$ on $t$ using the public key of $s P K_{s}$. \\
\hline$V_{i}$ & A mutation tuple with the form $\left\{p o s, L_{p o s}\right\}$. \\
\hline$C O M(x)$ & $\begin{array}{l}\text { A commitment scheme allowing zero-knowledge range proofs over } x . \\
\text { Equivalent to } C O M(x, s) \text { where } s \text { is a random bitstring used to } \\
\text { create the commitment. }\end{array}$ \\
\hline$\delta$ & $\begin{array}{l}\text { Expansion rate of a range proof. Calculated as } \frac{Y-X}{y-x} \\
\text { where }[x, y] \text { is range requested and }[X, Y] \text { is range proven the value to be in }\end{array}$ \\
\hline
\end{tabular}

dimensions. We then describe the proposed technique and argue its security, privacy, and efficiency.

Intuitive Approaches. One trivial solution is to use the full genome representation: at sequencing time, $S L$ signs all position-base pairs for each position in the DNA and later, when $T$ asks for all SNPs in a range, Alice provides all pairs in that range with their corresponding signatures. The tester can easily detect any missing positions. Inclusion of fake bases is impossible since Alice cannot generate signatures on behalf of $S L$. This approach provides authenticity and integrity and leaks no information about bases outside the queried range. Unfortunately, it has high computation and storage costs due to sheer size of the DNA. To reduce these costs, optimizations such as condensed and aggregated signatures can be applied, though the final cost would be still far from optimal. We refer the reader to [12] for a more detailed comparison of such methods.

Using a reference genome and representing the genome only in terms of mutations with respect to the reference genome (as in Section 3.1) reduces storage and computation costs substantially. However, it introduces the completeness problem. Suppose that $T$ queries for mutations in range $[x, y]$ where $x$ and $y$ are DNA positions. If each mutation is signed by the $S L$, Alice could reply with a list of authentic mutations in that range. However, this would not prevent Alice from excluding one or more mutations. To ensure sequential continuity of mutations, $S L$ could sign tuples consisting of two neighboring mutations sorted in ascending order, as suggested by [20]. However, this would entail revealing two tuples that contain mutations in positions immediately outside the lower and upper boundaries, respectively. Due to sparsity of genomic mutations, this could leak a substantial amount of sensitive information.

Proposed Approach. We assume that Alice receives from $S L$ a digital representation of her DNA in the form of a list of mutations:

$$
\mathcal{V}^{*}=\left\langle V_{-\infty}, V_{1}, V_{2}, \cdots, V_{n}, V_{+\infty}\right\rangle
$$

wrt the reference genome, where $V_{i} \in \mathcal{V}^{*}$ and for each $i, V_{i}$.pos < $V_{i+1}$.pos. Note that two special mutations, $V_{-\infty}\left(V_{-\infty} . p o s<0\right)$ and $V_{+\infty}\left(V_{+\infty}\right.$. pos $\left.>3.2 \cdot 10^{9}\right)$ are introduced to mark the lower and upper boundaries of the genome. Additionally, Alice receives a sequence of signatures $\gamma=\left\langle\gamma_{s}, \gamma_{s+1}, \cdots, \gamma_{s+n}\right\rangle$ where each $\gamma_{i}=$
$\operatorname{Sign}_{S K_{S L}}\left(T_{i}\right) . S K_{S L}$ is the secret key of $S L$ and the tuple $T_{i}$ is of the form:

$T_{i}=\left\{\operatorname{COM}\left(V_{i} \cdot p o s, s_{i_{1}}\right), \operatorname{COM}\left(V_{i}, s_{i_{2}}\right), \operatorname{COM}\left(V_{i+1} \cdot p o s, s_{i+1_{1}}\right), \operatorname{COM}\left(V_{i+1}, s_{i+1_{2}}\right)\right\}$

$C O M$ is a commitment scheme realizing zero-knowledge range queries. It uses a random binary string (e.g., $s_{i_{1}}$ ) as a salt to hide the committed value. Salts are re-used for the same mutation in another tuple (e.g., in $T_{i+1}$, commitment for $V_{i+1}$.pos and $V_{i+1}$ uses same salts $s_{i+1_{1}}$ and $s_{i+1_{2}}$, respectively).

Our scheme follows the model defined in Section 5. Resp $(Q)$ and $\operatorname{Verfy}(\mathcal{V}, \sigma)$ are presented in Figure 2. Note that, if either Verify () or ZK_Verify() fails, $\operatorname{Verfy}(\mathcal{V}, \sigma)$ terminates with output $\perp$. See Table 1 for description of verification functions.
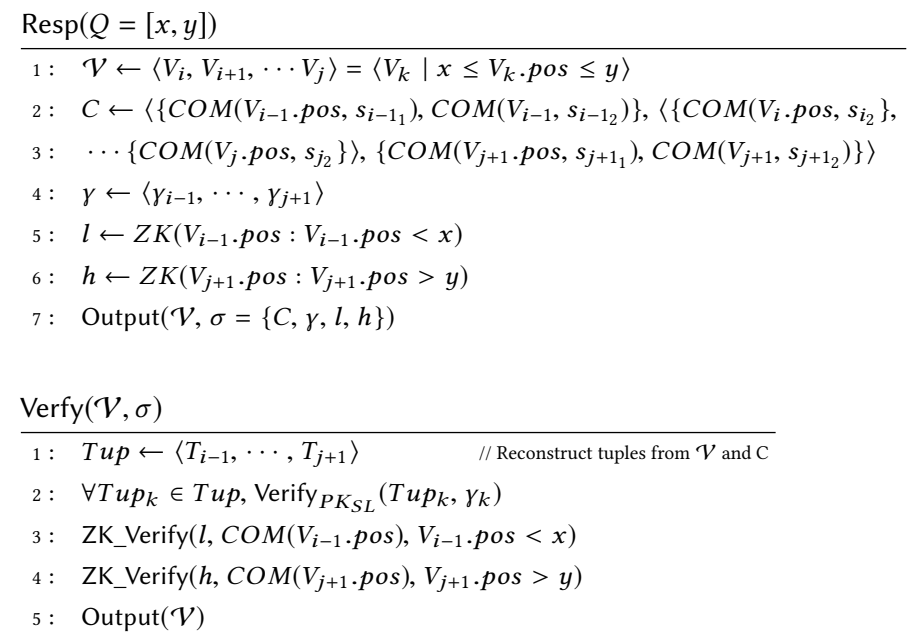

Figure 2: $\operatorname{Resp}(Q)$ and $\operatorname{Verfy}(\mathcal{V}, \sigma)$ functions.

\subsection{Security \& Privacy}

We now analyze security and privacy of the proposed construction, with respect to goals stated in Section 5. Suppose that $\mathcal{V}$ is nonempty. There are two tuples of the forms (salts are not shown here):

$$
\left\{\operatorname{COM}\left(V_{i-1} \cdot p o s\right), \operatorname{COM}\left(V_{i-1}\right), \operatorname{COM}\left(V_{i}\right), \operatorname{COM}\left(V_{i} \cdot p o s\right)\right\}
$$

and

$$
\left\{\operatorname{COM}\left(V_{j} \cdot p o s\right), \operatorname{COM}\left(V_{j}\right), \operatorname{COM}\left(V_{j+1}\right), \operatorname{COM}\left(V_{j+1} \cdot p o s\right)\right\}
$$

Alice first reveals the commitments to mutations in the range, allowing $T$ to validate the signatures. Then, Alice proves to $T$ in zero-knowledge:

(1) $V_{i-1}$.pos is out of range.

(2) $V_{j+1}$.pos is out of range.

As a result, $T$ is convinced that all other mutations in positions below $V_{i-1}$.pos and above $V_{j+1}$.pos are out of range. $T$ also confirms that $V_{i}$ and $V_{j}$ are in range by using the revealed values of both commitments. Note that $V_{i-1}$ and $V_{j+1}$ can be $V_{-\infty}$ and $V_{\infty}$ respectively. 
Suppose that $\mathcal{V}$ is empty. There is a tuple of the form:

$\left\{\operatorname{COM}\left(V_{l}\right.\right.$.pos $\left.), \operatorname{COM}\left(V_{l}\right), \operatorname{COM}\left(V_{l+1}\right), \operatorname{COM}\left(V_{l+1} \cdot p o s\right)\right\}$

where $V_{l}$.pos $<x$ and $V_{l+1}$.pos $>y$. Alice proves the inequality to $T$ in zero-knowledge.

Goal 1. Authenticity is ensured due to security of the underlying digital signature scheme used by $S L$ to sign tuples.

Goal 2. Completeness is achieved using sequential linking of elements (mutation tuples) thus allowing $T$ to detect any exclusion. Also, based on Goal 1, it is impossible for Alice to introduce any additional mutations.

Goal 3. Due to the use of zero-knowledge proofs and hiding commitments, no information about positions of any out-of-range mutation is revealed.

One special case occurs when $V_{i-1}$ and $V_{l}$ (in Equations 1 and 3) and $V_{j+1}$ and $V_{l+1}$ (in Equations 2 and 3) are: $V_{-\infty}$ and $V_{\infty}$, respectively. Such sentinel commitments should be indistinguishable from other commitments. However, if needed to be revealed, they should clearly denote genome boundaries.

This can be achieved by using positions 0 and $3.2 \cdot 10^{9}+1$ as $V_{-\infty}$ and $V_{+\infty}$ commitments, respectively, and allowing actual mutation positions to start from 1.

\subsection{Instantiation of Proposed Construction}

As an example commitment scheme $C O M$, we use Fujisaki-Okamoto commitments [24]. For range proofs we use Boudot's technique [11] with expansion rate $\delta=1$. Less than and greater than are implemented as follows:

To show that a value $v$ is less than $x$ in zero-knowledge, the range proof convinces the verifier that $v$ in commitment $\operatorname{COM}(v)$ is in range [ $\min -1, x-1]$, where min is the smallest possible value of $v$. Similarly, for greater-than proofs, the range proof convinces the verifier that $v \in[x+1, \max +1]$, where $\max$ is the maximum possible value of $v$. In the genomic context, $\max =3.2 \times 10^{9}$ and $\min =1$.

\section{EFFICIENCY CONSIDERATIONS}

\subsection{Commitments and Salt Generation}

Since Fujisaki-Okamoto commitments are expensive due to modular exponentiation operations, we use the SHA2 hash function with a large salt for mutation commitments. Depending on storage requirements, salt generation can be done using a key derivation function, such as HKDF [29], or a Pseudo Random Number Generator (PRNG) with a sufficiently large seed. This would reduce the storage cost of salts.

We construct a tuple as:

$\left\{F O\left(V_{i}\right.\right.$. pos $\left.\left.), \operatorname{SHA} 2\left(V_{i}, s_{i}\right), F O\left(V_{i+1} . p o s\right), \operatorname{SHA} 2\left(V_{i+1}, s_{i+1}\right)\right)\right\}$ where $F O$ is the Fujisaki-Okamoto commitment and $s_{i}$ is a 128-bit salt.

\subsection{Signatures}

To provide authenticity, each tuple described in Section 7.1 is signed by $S L$ at the initial sequencing time. To reduce signature sizes, we use EC-DSA with the elliptic curve secp256r1, which yields 512-bit signatures.

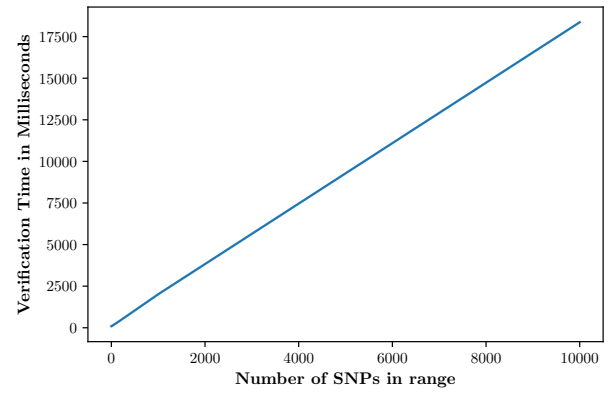

Figure 3: Verification times for $T$ given number of SNPs in range

\section{EVALUATION}

We have implemented a prototype in Java on a PC with an Intel(R) Core(TM) i7-3770 CPU @ 3.40GHz chip and 16GB of RAM. We use the code from [4] to implement commitments and proofs realizing range queries, and Bouncy Castle [1] for other crypto primitives. For Fujisaki-Okamoto commitments we use the following parameters: $s=552$ while $t$ and $l$ in the range proofs are 128 and 40 respectively.

Since genomic mutations are distributed at every 1,000 bases [3] in average, pre-processing includes $3 \times 10^{6}$ mutations. The entire offline phase (performed by $S L$ ) takes about 4.2 hours on the aforementioned platform. Specifically, $S L$ needs to compute one signature, one range proof commitment and one SHA2 commitment per mutation. In the online phase, $T$ makes $r+1$ signature verifications ( $r$ - \# mutations in range) and 2 range proof verifications while Alice generates two range proofs. We measure the CPU time spent for the main cryptographic operations using our Java prototype. The results are reported in Table 2.

Table 2: Measured computation costs of cryptographic operations.

\begin{tabular}{|l|l|}
\hline Computations & Time (in milliseconds) \\
\hline Fujisaki-Okamoto commitment: & 3.5 \\
\hline Zero-knowledge proof generation: & 47.7 \\
\hline Proof validation & 37 \\
\hline SHA256 commitment & $\begin{array}{l}0.3 \text { (including salt creation using Java's SecureRandom } \\
\text { class [2] }\end{array}$ \\
\hline SHA256 commitment validation & 0.1 \\
\hline
\end{tabular}

Verification cost performed by $T$ scales linearly with the number of SNPs in the queried range (as shown in Figure 3), and range proof verification cost is dominated by the signature verification cost.

\section{CONCLUSION}

While genomic privacy has attracted much attention due to the dire consequences of possible leaks, insufficient attention is paid to genomic security, i.e., authentication and integrity of genomic data. This paper proposes a cryptographic technique for efficient, secure and private genomic range queries. It ensures the unforgeability and completeness of genomic material needed by the tester from the user, and also protect user privacy as it leaks no extra information to the tester than necessary. 


\section{REFERENCES}

[1] [n. d.]. The Legion of the Bouncy Castle. https://www.bouncycastle.org/. ([n d.]). Accessed: 2019-02-12.

[2] [n. d.]. SecureRandom (Java Platform SE 8 ). https://docs.oracle.com/javase/8/ docs/api/java/security/SecureRandom.html. ([n. d.]). Accessed: 2019-02-13.

[3] [n. d.]. What are single nucleotide polymorphisms (SNPs)? https://ghr.nlm.nih gov/primer/genomicresearch/snp. ([n. d.]). Accessed: 2019-02-05.

[4] [n. d.]. Zero-Knowledge Proofs. https://github.com/ing-bank/zkproofs. ([n. d.]) Accessed: 2019-02-12.

[5] Mete Akgün, A Osman Bayrak, Bugra Ozer, and M Şamil Sağıroğlu. 2015. Privacy preserving processing of genomic data: A survey. Fournal of biomedical informatics 56 (2015), 103-111.

[6] Mikhail J Atallah, Florian Kerschbaum, and Wenliang Du. 2003. Secure and private sequence comparisons. In Proceedings of the 2003 ACM workshop on Privacy in the electronic society. ACM, 39-44.

[7] Mikhail J Atallah and Jiangtao Li. 2005. Secure outsourcing of sequence comparisons. International fournal of Information Security 4, 4 (2005), 277-287.

[8] Erman Ayday, Jean Louis Raisaro, and Jean-Pierre Hubaux. 2012. Privacyenhancing technologies for medical tests using genomic data. Technical Report.

[9] Erman Ayday, Jean Louis Raisaro, Jean-Pierre Hubaux, and Jacques Rougemont. 2013. Protecting and evaluating genomic privacy in medical tests and personalized medicine. In Proceedings of the 12th ACM workshop on Workshop on privacy in the electronic society. ACM, 95-106.

[10] Pierre Baldi, Roberta Baronio, Emiliano De Cristofaro, Paolo Gasti, and Gene Tsudik. 2011. Countering gattaca: efficient and secure testing of fully-sequenced human genomes. In Proceedings of the 18th ACM conference on Computer and communications security. ACM, 691-702.

[11] Fabrice Boudot. 2000. Efficient proofs that a committed number lies in an interval. In International Conference on the Theory and Applications of Cryptographic Techniques. Springer, 431-444.

[12] Tatiana Bradley, Xuhua Ding, and Gene Tsudik. 2017. Genomic security (lest we forget). IEEE Security \& Privacy 15, 5 (2017), 38-46.

[13] Ernest F Brickell, David Chaum, Ivan B Damgård, and Jeroen van de Graaf. 1987. Gradual and verifiable release of a secret. In Conference on the Theory and Application of Cryptographic Techniques. Springer, 156-166.

[14] A Chan, Y Frankel, and Y Tsiounis. 1998. Easy come-easy go divisible cash. updated version with corrections. Technical Report. GTE Tech. Rep.

[15] George Danezis and Emiliano De Cristofaro. 2014. Fast and private genomic testing for disease susceptibility. In Proceedings of the 13th Workshop on Privacy in the Electronic Society. ACM, 31-34.

[16] Emiliano De Cristofaro, Sky Faber, Paolo Gasti, and Gene Tsudik. 2012. Genodroid: are privacy-preserving genomic tests ready for prime time?. In Proceedings of the 2012 ACM workshop on Privacy in the electronic society. ACM, 97-108.

[17] Emiliano De Cristofaro, Sky Faber, and Gene Tsudik. 2013. Secure genomic testing with size-and position-hiding private substring matching. In Proceedings of the 12th ACM workshop on Workshop on privacy in the electronic society. ACM, 107-118.

[18] Emiliano De Cristofaro, Jihye Kim, and Gene Tsudik. 2010. Linear-complexity private set intersection protocols secure in malicious model. In International Conference on the Theory and Application of Cryptology and Information Security. Springer, 213-231.

[19] Emiliano De Cristofaro and Gene Tsudik. 2010. Practical private set intersection protocols with linear complexity. In International Conference on Financial Cryptography and Data Security. Springer, 143-159.

[20] Premkumar Devanbu, Michael Gertz, Charles Martel, and Stuart G Stubblebine 2003. Authentic data publication over the Internet 1. Fournal of Computer Security 11, 3 (2003), 291-314.

[21] Tamara Dugan and Xukai Zou. 2016. A Survey of Secure Multiparty Computation Protocols for Privacy Preserving Genetic Tests. In Connected Health: Applications, Systems and Engineering Technologies (CHASE), 2016 IEEE First International Conference on. IEEE, 173-182.

[22] Yanxiao Feng, Yuechuan Zhang, Cuifeng Ying, Deqiang Wang, and Chunlei Du. 2015. Nanopore-based fourth-generation DNA sequencing technology. Genomics, proteomics \& bioinformatics 13, 1 (2015), 4-16.

[23] Michael J Freedman, Kobbi Nissim, and Benny Pinkas. 2004. Efficient private matching and set intersection. In International conference on the theory and applications of cryptographic techniques. Springer, 1-19.

[24] Eiichiro Fujisaki and Tatsuaki Okamoto. 1997. Statistical zero knowledge protocols to prove modular polynomial relations. In Annual International Cryptology Conference. Springer, 16-30.

[25] Stephanie J Heerema and Cees Dekker. 2016. Graphene nanodevices for DNA sequencing. Nature nanotechnology 11, 2 (2016), 127.

[26] Bijit Hore, Sharad Mehrotra, and Gene Tsudik. 2004. A privacy-preserving index for range queries. In Proceedings of the Thirtieth international conference on Very large data bases-Volume 30. VLDB Endowment, 720-731.

[27] Stanisław Jarecki and Xiaomin Liu. 2010. Fast secure computation of set intersection. In International Conference on Security and Cryptography for Networks.
Springer, 418-435

[28] Murat Kantarcioglu, Wei Jiang, Ying Liu, and Bradley Malin. 2008. A cryptographic approach to securely share and query genomic sequences. IEEE Transactions on information technology in biomedicine 12, 5 (2008), 606-617.

[29] Hugo Krawczyk. 2010. Cryptographic extraction and key derivation: The HKDF scheme. In Annual Cryptology Conference. Springer, 631-648.

[30] Feifei Li, Marios Hadjieleftheriou, George Kollios, and Leonid Reyzin. 2006. Dynamic authenticated index structures for outsourced databases. In Proceedings of the 2006 ACM SIGMOD international conference on Management of data. ACM, 121-132.

[31] Wenbo Mao. 1998. Guaranteed correct sharing of integer factorization with off-line shareholders. In International Workshop on Public Key Cryptography. Springer, 60-71.

[32] Elaine R Mardis. 2011. A decadeâĂŹs perspective on DNA sequencing technology. Nature 470, 7333 (2011), 198.

[33] Muhammad Naveed, Erman Ayday, Ellen W Clayton, Jacques Fellay, Carl A Gunter, Jean-Pierre Hubaux, Bradley A Malin, and XiaoFeng Wang. 2015. Privacy in the genomic era. ACM Computing Surveys (CSUR) 48, 1 (2015), 6.

[34] HweeHwa Pang, Arpit Jain, Krithi Ramamritham, and Kian-Lee Tan. 2005. Verifying completeness of relational query results in data publishing. In Proceedings of the 2005 ACM SIGMOD international conference on Management of data. ACM, 407-418.

[35] Juan Ramón Troncoso-Pastoriza, Stefan Katzenbeisser, and Mehmet Celik. 2007. Privacy preserving error resilient DNA searching through oblivious automata. In Proceedings of the 14th ACM conference on Computer and communications security. ACM, 519-528. 